\title{
ONLINE TRACKING OF THE DEGREE OF NONLINEARITY WITHIN COMPLEX SIGNALS
}

\author{
${ }^{1}$ Danilo P. Mandic, ${ }^{1}$ Phebe Vayanos, ${ }^{1}$ Soroush Javidi, ${ }^{1}$ Beth Jelfs and ${ }^{2}$ Kazuyuki Aihara \\ ${ }^{1}$ Imperial College London, ${ }^{2}$ University of Tokyo \\ \{d.mandic, beth.jelfs, soroush.javidi, foivi.vayanos\}@imperial.ac.uk, aihara@sat.t.u-tokyo.ac.jp
}

\begin{abstract}
A novel method for online tracking of the changes in the nonlinearity within complex-valued signals is introduced. This is achieved by a collaborative adaptive signal processing approach by means of a hybrid filter. By tracking the dynamics of the adaptive mixing parameter within the employed hybrid filtering architecture, we show that it is possible to quantify the degree of nonlinearity within complex-valued data. Simulations on both benchmark and real world data support the approach.
\end{abstract}

Index Terms - Adaptive signal processing, complex LMS, convex optimisation, machine learning, wind modelling.

\section{INTRODUCTION}

Signal modality characterisation reveals the changes in the nature of real world data (degree of sparsity, or nonlinearity) and is very important in online applications. Some aspects of this problem for the analysis of EEG data have been addressed in [1]. Another benefit of tracking the degree of linearity in a signal in real-time, is for example, to provide prior knowledge to a blind algorithm. The degree of nonlinearity can be used as a signal "fingerprint", and when combined with other signal modality trackers [2] this can be a powerful tool for machine learning, detailing changes within the signal dynamics along time.

We propose to achieve this by a collaborative signal processing approach; by means of hybrid filters [3], a convex mixing parameter within this structure is updated in an online manner in order to illustrate the modality change of the signal in hand. It is, however, much more complicated to achieve the tracking of modality change in the complex domain $\mathbb{C}$. The extensions of hybrid filters from $\mathbb{R}$ to $\mathbb{C}$ are non-trivial, this is due to the fact that the nature of nonlinearity in $\mathbb{C}$ is fundamentally different from that in $\mathbb{R}$. For instance, in the design of learning algorithms, it should be taken into account that the only continuously differentiable function in $\mathbb{C}$ is a constant (Liouville's theorem).

A first attempt to track the modality change of complex signals using hybrid filters was introduced in [2], where the changes between the split- and fully-complex natures of the data were tracked. The nonlinearity of the input signal was implicitly assumed, this however, may not necessarily be the case. Thus, before checking for the split- or fully-complex nonlinear na- ture, we first need to assess whether the input is linear or nonlinear.

Our underlying idea is to use a technique similar to that in [4], and construct a hybrid filter in $\mathbb{C}$ whereby each subfilter is designed so as to perform best, on either linear or nonlinear input. By making these subfilters collaborate and by tracking the values of the mixing parameter, we can then assess the degree of nonlinearity.

Complex-valued signals are either complex by design or are made complex by convenience of representation. An example of a real-valued signal which is best analysed in $\mathbb{C}$ is wind, where the fusion of the speed and direction creates a single complex-valued wind signal.

We shall first describe the hybrid filter configuration and derive the update for the mixing parameter. Next, the Complex Nonlinear Gradient Descent (CNGD) and Complex Least Mean Square (CLMS) algorithms and their normalised variants are briefly introduced. Finally, the performance of the method is assessed using benchmark linear and nonlinear signals, as well as a complex-valued wind signal.

\section{HYBRID FILTER CONFIGURATION}

A hybrid filter, shown in Figure 1, consist of two subfilters, each being adapted independently. A convex combination of the two filters is then taken as the output of the hybrid filter. Since a convex combination $z$ of two points $x$ and $y$ is defined as $\lambda x+(1-\lambda) y, \lambda \in[0,1]$ (shown in Figure 2), the values of $\lambda$ will indicate which of the subfilters is better suited to the nature of the input.

The two subfilters within the hybrid filtering architecture operate in the prediction setting, sharing the complex input $x(k)$. The convex combination of the subfilter outputs $y_{\text {nonlinear }}(k)$ and $y_{\text {linear }}(k)$ forms the overall system output $y(k)$, given by

$$
y(k)=\lambda(k) y_{\text {nonlinear }}(k)+(1-\lambda(k)) y_{\text {linear }}(k)
$$

where $\lambda(k)$ is the mixing parameter, which is made adaptive, and is updated by minimising the cost function

$$
E(k)=\frac{1}{2}|e(k)|^{2}=\frac{1}{2}|d(k)-y(k)|^{2}
$$

We can obtain the update for $\lambda(k)$ using a stochastic gradient based adaptation, such as the LMS, whereby

$$
\lambda(k+1)=\lambda(k)-\left.\mu_{\lambda} \nabla_{\lambda} E(k)\right|_{\lambda=\lambda(k)}
$$




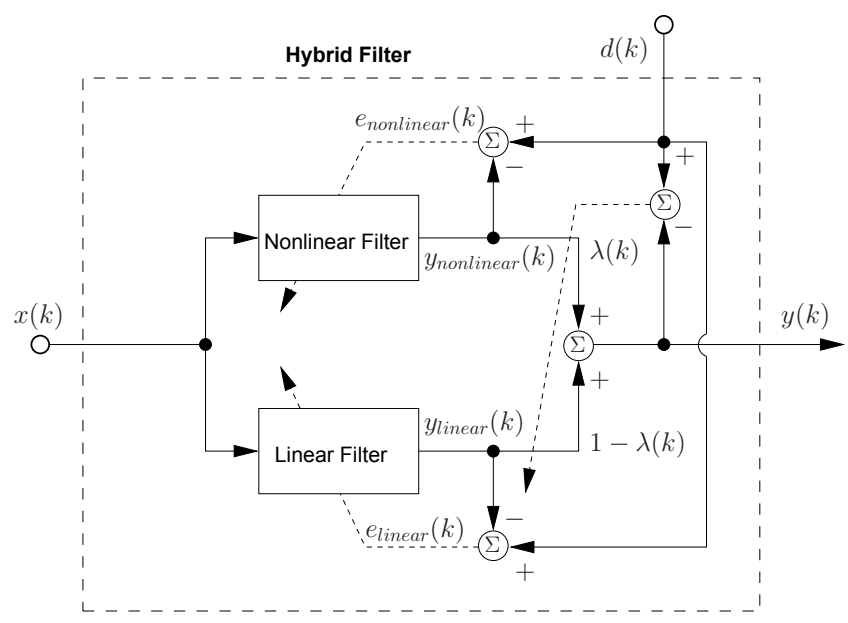

Fig. 1. Hybrid filter with complex input $x(k)$, consisting of a linear and nonlinear subfilter.

and $\mu_{\lambda}$ is the step size. It is noted that since the input to the filters is complex, the error $e(k)$ is also complex, and therefore

$$
\left.\nabla_{\lambda} E(k)\right|_{\lambda=\lambda(k)}=\left\{e(k) \frac{\partial e^{*}(k)}{\partial \lambda(k)}+e^{*}(k) \frac{\partial e(k)}{\partial \lambda(k)}\right\}
$$

The two gradient terms from (4) can be evaluated as

$$
\begin{aligned}
\frac{\partial e(k)}{\partial \lambda(k)} & =\frac{\partial e_{r}(k)}{\partial \lambda(k)}+\jmath \frac{\partial e_{i}(k)}{\partial \lambda(k)} \\
\frac{\partial e^{*}(k)}{\partial \lambda(k)} & =\frac{\partial e_{r}(k)}{\partial \lambda(k)}-\jmath \frac{\partial e_{i}(k)}{\partial \lambda(k)}
\end{aligned}
$$

where $(\cdot)_{r}$ and $(\cdot)_{i}$ denote respectively the real and imaginary part of a complex number. Rewriting (1) in terms of its real and imaginary part and substituting into (2) yields

$$
\begin{aligned}
\frac{\partial e(k)}{\partial \lambda(k)} & =y_{\text {linear }}(k)-y_{\text {nonlinear }}(k) \\
\frac{\partial e^{*}(k)}{\partial \lambda(k)} & =\left(y_{\text {linear }}(k)-y_{\text {nonlinear }}(k)\right)^{*}
\end{aligned}
$$

Finally, the gradient (4) becomes

$$
\left.\nabla_{\lambda} E(k)\right|_{\lambda=\lambda(k)}=\Re\left\{e(k)\left(y_{\text {linear }}(k)-y_{\text {nonlinear }}(k)\right)^{*}\right\}
$$

where $\Re(\cdot)$ denotes the real part of a complex number, which yields the mixing parameter update as

$$
\lambda(k+1)=\lambda(k)+\mu_{\lambda} \Re\left\{e(k)\left(y_{\text {nonlinear }}(k)-y_{\text {linear }}(k)\right)^{*}\right\}
$$

\subsection{Learning Algorithms}

While standard hybrid filters aim to provide best output performance based on the convex combination of the subfilters,

\section{x $\quad \lambda x+(1-\lambda) y \quad$ y}

Fig. 2. Convex combination of two points $x$ and $y$.

our aim is to solely track the variation of $\lambda(k)$. To that end, the nonlinear and linear subfilter of Figure 1 are adapted using the CNGD [5] and CLMS [6], respectively. Their normalised variants CNNGD ans NCLMS will also be used.

The linear CLMS update is given by

$$
\begin{aligned}
y_{\text {linear }}(k) & =\mathbf{x}^{T}(k) \mathbf{w}_{\text {linear }}(k) \\
e_{\text {linear }}(k) & =d(k)-y_{\text {linear }}(k) \\
\mathbf{w}_{\text {linear }}(k+1) & =\mathbf{w}_{\text {linear }}(k)+\mu e_{\text {linear }}(k) \mathbf{x}^{*}(k)
\end{aligned}
$$

whereas the update for the adaptation of the nonlinear subfilter CNNGD is given by

$$
\begin{aligned}
y_{\text {nonlinear }}(k) & =\Phi(\underbrace{\mathbf{x}^{T}(k) \mathbf{w}_{\text {nonlinear }}(k)}_{\text {net }(k)}) \\
e_{\text {nonlinear }}(k) & =d(k)-y_{\text {nonlinear }}(k) \\
\mathbf{w}_{\text {nonlinear }}(k+1) & =\mathbf{w}_{\text {nonlinear }}(k) \\
& +\eta e_{\text {nonlinear }}(k)\left[\Phi^{\prime}(\operatorname{net}(k))\right]^{*} \mathbf{x}^{*}(k)
\end{aligned}
$$

The normalised variants, NCLMS and CNNGD, are specified by

$$
\begin{aligned}
& \mu_{N C L M S}=\mu /\left(\|\mathbf{x}(k)\|_{2}^{2}+\epsilon\right) \\
& \eta_{C N N G D}=\eta /\left(\left[\Phi^{\prime}(\operatorname{net}(k))\right]^{2}\|\mathbf{x}\|_{2}^{2}+\epsilon\right)
\end{aligned}
$$

\section{TRACKING OF NONLINEARITY WITHIN COMPLEX SIGNALS}

For generality, two sets of synthesised benchmark signals and a real-world complex wind dataset were used in simulations. The linear processes considered were a stable complex autoregressive $A R(2)$ and $A R(4)$, given respectively by

$$
x(k)=0.9 x(k-1)+n(k)
$$

and

$$
\begin{aligned}
x(k) & =1.79 x(k-1)-1.85 x(k-2)+1.27 x(k-3) \\
& -0.41 x(k-4)+n(k)
\end{aligned}
$$

where $n(k)=n_{r}(k)+\jmath n_{i}(k)$ is a complex white Gaussian noise $(\mathrm{CWGN})$, for which the real and imaginary parts are independent real WGN sequences $\sim \mathcal{N}(0,1)$ and $\sigma_{n}^{2}=\sigma_{n_{r}}^{2}+$ $\sigma_{n_{i}}^{2}$

As nonlinear complex signals, we used benchmark [7]

$$
x(k)=\frac{x(k-1)}{1+x^{2}(k-1)}+n^{3}(k)
$$


and

$$
x(k)=\frac{x^{2}(k-1)(x(k-1)+2.5)}{1+x^{2}(k-1)+x^{2}(k-2)}+n(k-1)
$$

To illustrate the ability of the hybrid filter to track the modality changes within a signal, experiments were performed on alternating sequences of linear ((13) or (14)) and nonlinear ((15) or (16)) data. An additional set of experiments was conducted on a set of real-world wind data ${ }^{1}$. For all simulations, the initial weight vectors for both filters were set to zero and the filter order was $N=10$. When a nonlinear CNGD or CNNGD was used, the nonlinearity at the output of the filter was the complex logistic function

$$
\Phi(z)=1 /\left(1+e^{-z}\right)
$$

\subsection{Combination of CNGD and CLMS}

In the first set of experiments, the nature of the input alternated every 200 samples between linear and nonlinear. The evolution of $\lambda$ is shown in Figure 3 for two different settings. In both cases, $\mu_{C N G D}$ was set equal to $\mu_{C L M S}$, and it was always possible to detect both the direction of the change from linear to nonlinear and vice versa, and the degree of such change, as illustrated by the values of $\lambda$ approaching 0.85 for nonlinear data and 0.1 and 0.4 for linear data. Also, this approach was robust to changes in the relative values of $\mu_{C N G D}$ and $\mu_{C L M S}$.

\subsection{Combination of CNNGD and CNLMS}

Next in order to overcome some problems with signal conditioning, CNNGD (12) and CNLMS (11) were combined in a hybrid fashion. The results shown in Figure 4 demonstrate the robustness of this combination compared to that from Figure 3. Indeed, by setting $\mu_{C N N G D}=\mu_{C N L M S}=0.8$, the hybrid filter performed well on a range of synthetically generated signals and accurately detected both the direction of the change from linear to nonlinear and vice versa, and the degree of the change. Furthermore, it can be seen that as the two transversal filters converge, the tracking of the degree of nonlinearity in the input is improved in terms of the range swept by $\lambda$ (due to learning).

Next, the experiments were performed on complex-valued wind data, and the simulation results are shown in Figure 5. The combination of CNNGD and CNLMS was clearly capable of tracking changes in the linear/nonlinear nature of the intermittent and nonstationary wind. The wind was changing its nature in the region between (1-5000) and (10000-15000) samples, which was correctly reflected in the values of $\lambda$. For steady wind, the nature of wind exhibited a medium degrees of nonlinearity.

In conclusion, the combination of CNNGD and CNLMS provided excellent results in the identification of signal noninearity on a broad range of inputs, provided the individual filters converged.

\footnotetext{
${ }^{1}$ The wind data with speed $v$ and direction $\varphi$ were made complex as $\mathbf{v}=$ $v e^{i \varphi}[5]$
}

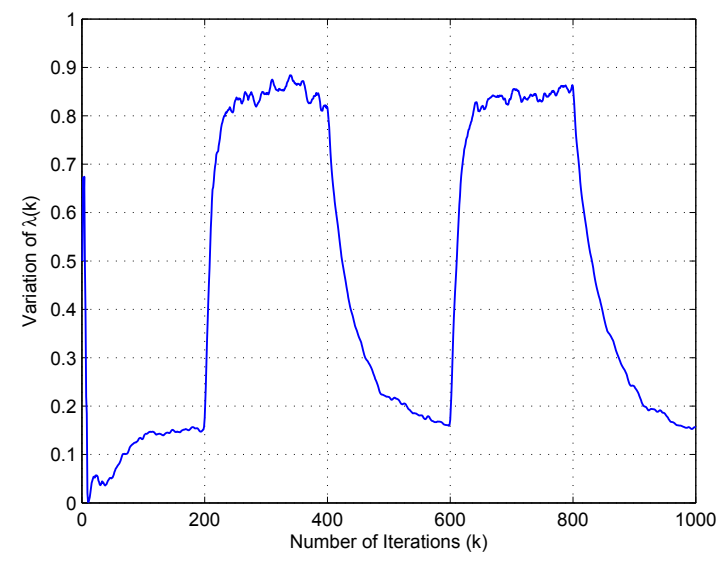

(a) Variation of the mixing parameter $\lambda$ for the alternating inputs (13) and (15) $\left(\mu_{C N G D}=0.08, \mu_{C L M S}=0.08, \mu_{\lambda}=50\right)$

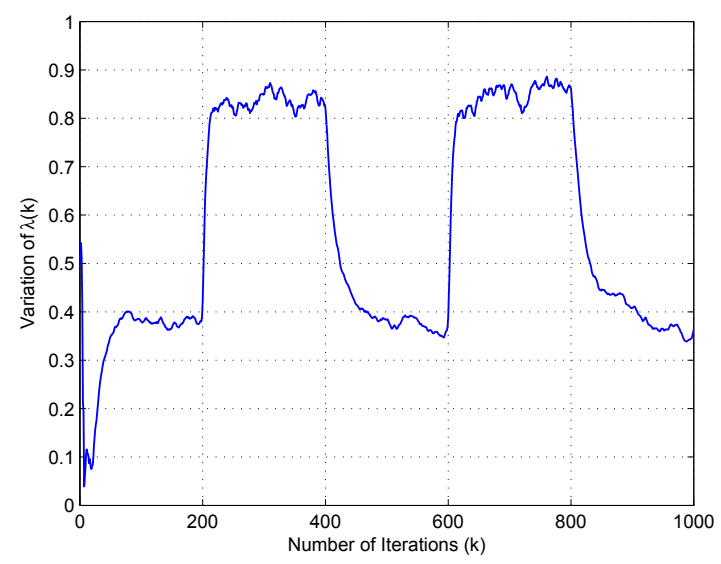

(b) Variation of the mixing parameter $\lambda$ for the alternating inputs (14) and (16) $\left(\mu_{C N G D}=0.2, \mu_{C L M S}=0.2, \mu_{\lambda}=60\right)$

Fig. 3. Hybrid combination of CNGD and CLMS, for input natures alternating between linear and nonlinear every 200 samples

\section{CONCLUSION}

We have introduced a method for online tracking of signal nonlinearity in the complex domain, which not only reveals the signal nature but it also can assist in machine learning applications. This has been achieved by using a collaborative signal processing approach, whereby the subfilters within a hybrid filter were adapted using the CLMS and CNGD algorithms and their normalised variants. Simulation results have shown that the proposed approach is capable of tracking the nonlinearity within both synthesised and real-world complex-valued data.

\section{REFERENCES}

[1] D.P Mandic, M Golz, A Kuh, D Obradovic, and T Tanaka, Eds., Signal Processing Techniques for Knowl- 


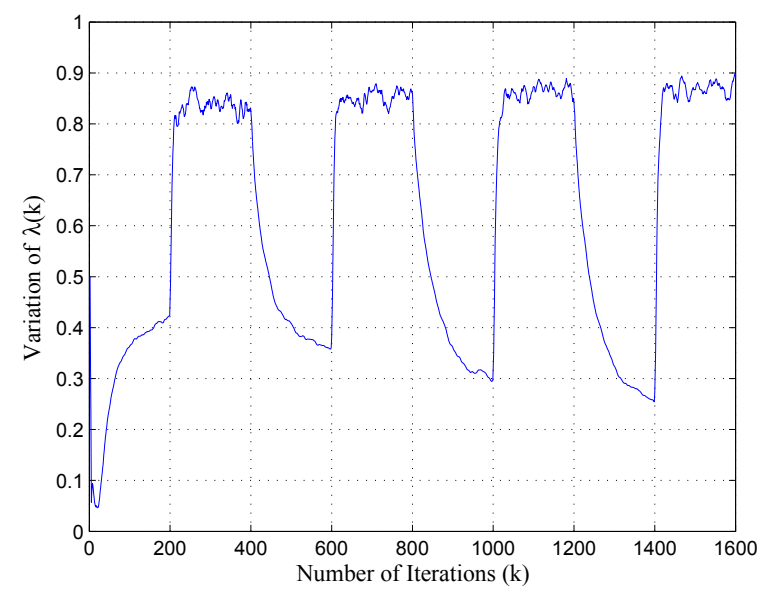

(a) Input nature alternating between linear (13) and nonlinear (15) $\left(\mu_{C N N G D}=0.8, \mu_{C N L M S}=0.8, \mu_{\lambda}=60\right)$

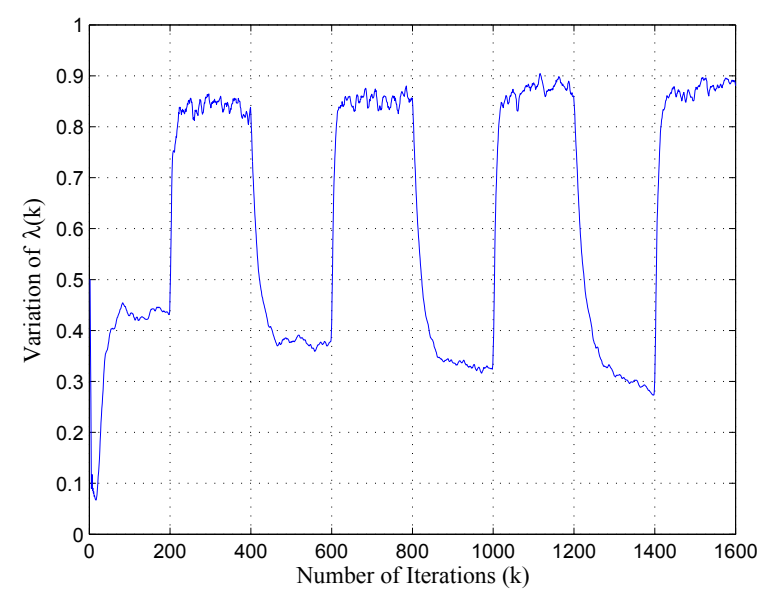

(b) Input nature alternating between linear (14) and nonlinear (16) $\left(\mu_{C N N G D}=0.8, \mu_{C N L M S}=0.8, \mu_{\lambda}=60\right)$

Fig. 4. Mixing parameter $\lambda$ at the output of the hybrid combination of CNNGD and CNLMS, for input nature alternating between linear and nonlinear every 200 samples

edge Extraction and Information Fusion, Springer, 2007.

[2] P. Vayanos, S.L. Goh, and D.P. Mandic, "Online detection of the nature of complex-valued signals," in Proceedings of the 16th IEEE Signal Processing Society Workshop on Machine Learning for Signal Processing, 2006, pp. 173-178.

[3] J. Arenas-Garcia, A.R. Figueiras-Vidal, and A.H. Sayed, "Mean-square performance of a convex combination of two adaptive filters," IEEE Transactions on Signal Processing, vol. 54, no. 3, pp. 1078-1090, 2006.

[4] D. Mandic, P. Vayanos, C. Boukis, B. Jelfs, S.L. Goh, T. Gautama, and T. Rutkowski, "Collaborative adaptive
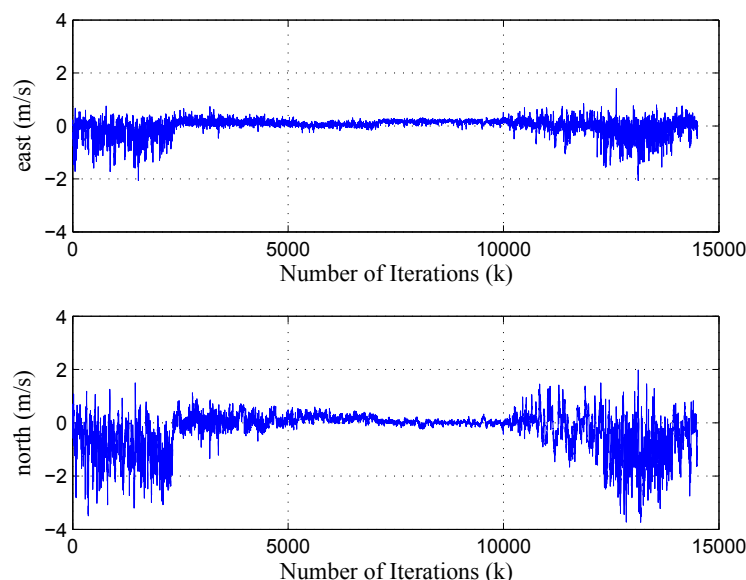

(a) The real and imaginary parts of complex-valued wind

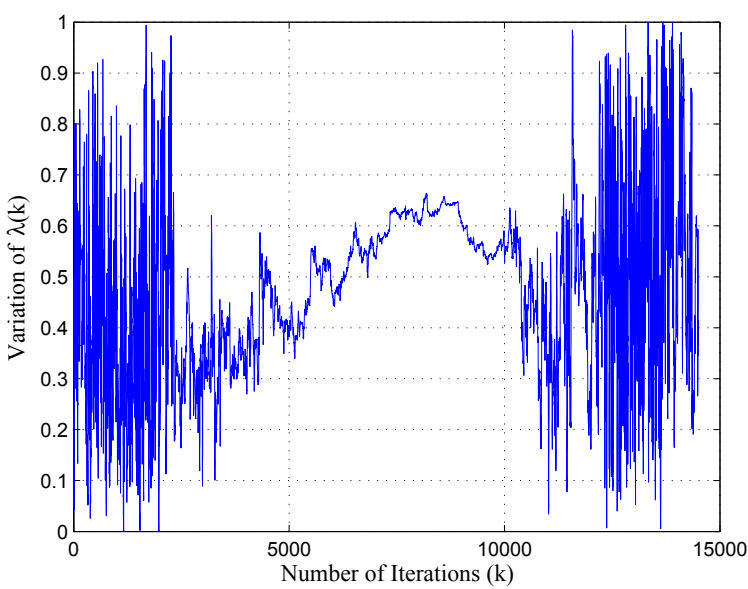

(b) Evolution of $\lambda$ for complex wind

Fig. 5. Evolution of $\lambda$ in the hybrid combination of $\mathrm{CN}$ NGD and CNLMS for the wind data set, for $\mu_{C N N G D}=0.8$, $\mu_{C N L M S}=0.8$ and $\mu_{\lambda}=60$.

learning using hybrid filters," in ICASSP 2007, 2007, vol. 3, pp. 921-924.

[5] D.P. Mandic and S.L. Goh, Complex Valued Nonlinear Adaptive Filters: A Neural Network Approach, John Wiley, 2008 (to be published).

[6] B. Widrow, J. McCool, and M. Ball, "The complex LMS algorithm," Proceedings of the IEEE, vol. 63, no. 4, pp. 719-720, 1975.

[7] K.S. Narendra and K. Parthasarathy, "Identification and control of dynamical systems using neural networks," IEEE Transactions on Neural Networks, vol. 1, no. 1, pp. 4-27, 1990. 